\title{
Creating a LGBTQ+ Inclusive Culture in the Athletic Training Facility
}

Sean M. Rogers, DAT, ATC ${ }^{1}$; Ashley K. Crossway, DAT, ATC ${ }^{2}$; Patricia A. Aronson, PhD, LAT, ATC ${ }^{3}$

'California State University, Northridge, Northridge, CA; ${ }^{2}$ Nazareth College, Rochester, NY; ${ }^{3}$ Lynchburg College, Lynchburg, VA

\section{Key Phrases}

Emotional Wellness and Mental Health, Pre-

Participation Exams and Screening, Cultural

Competency, Health Care Disparities

\section{Correspondence}

Dr. Sean Rogers, California State University, Northridge

Department of Kinesiology, 18111 Nordhoff Street,

Northridge, CA $91330-8287$.

E-mail: sean.rogers@csun.edu

Twitter: @DATsearog

\section{Full Citation}

Rogers SM, Crossway AK, Aronson PA. Creating a LGBTQ+ inclusive culture in the athletic training facility. Clin Pract Athl Train. 2018;1(1):11-14.

https://doi.org/10.31622/2018/0001.3

Submitted: May 15, 2018 Accepted: May 25, 2018

\section{COMMENTARY}

Lesbian, gay, bisexual, transgender, and queer

individuals often fall under the umbrella acronym LGBTQ+ and are represented as a community. However, each individual letter represents a distinct population with its own identity, challenges, and unique obstacles with regards to equity. ${ }^{1}$ In recent years, there has been significant advancements in civil rights legislation and overall social acceptance of the LGBTQ+ community. Hallmark legislative victories including the right to marriage ${ }^{2}$ and to openly serve in the Armed Forces $^{3}$ have resulted in long overdue legal protections for the LGBTQ+ community. Despite these advances, LGBTQ+ individuals still disproportionately suffer from health disparities arising from stigmatization, oppression, and discrimination. ${ }^{4,5}$ One significant societal barrier still adversely affecting the healthcare of LGBTQ+ individuals is the lack of healthcare providers that are both knowledgeable and capable of delivering culturally competent and inclusive patient care. ${ }^{5}$ Creating an inclusive environment welcoming LGBTQ+ individuals in the clinical setting is vital to healthcare of those within the community. This paper serves to address inclusion of LGBTQ+ patients through patientcentered care in the athletic training facility. Current literature suggests that one of the most predominant health disparities among the LGBTQ+ community is dissatisfaction and avoidance of healthcare as a whole, which directly leads to suboptimal patient outcomes. 5,6 Recent trends in healthcare promote practitioners utilizing a patient- centered approach focusing on the identity of a patient, and how it relates to overall Health Related Quality of Life(HRQOL). ${ }^{7}$ Adherence to this approach better serves and respects the identity of patient populations. 7,8 The theoretical framework of contemporary disablement models, such as the Nagi Model, challenges clinicians to simultaneously address multiple aspects of health and wellbeing for overall improved patient outcomes. ${ }^{9}$ In order for healthcare practitioners to provide quality patient care, and achieve appropriate patient outcomes for individuals of many diverse backgrounds, cultures, and identities, it is imperative to use a patient-centered approach.

\section{The Importance of Identity and Patient- Centered Care}

Utilizing a patient-centered approach requires the clinician to consider the patient's identity and how it contributes to the overall disability and societal implication of the injury or pathology. 7,8 The identity of LGBTQ+ patients, as with all patients, is multidimensional and includes the interests, goals, dreams, and desires derived from the intricacies of patients' past experiences. Utilization of a disablement model in conjunction with an understanding of a patient identity helps the practitioner create targeted interventions to best address the etiology of the injury or illness being treated. This practice in healthcare is what is known as patient- centered care. Patient-centered care is an approach to healthcare that focuses on the unique qualities of each individual patient. 7,8 
This approach to healthcare is driven by active patient participation and less by cliniciandominated dialogues.7,8 The patient-centered approach creates a clinician-patient relationship that is rooted in trust, empathy, understanding, and collaboration with respect to the patient's identity. ${ }^{7,8}$ In practice, a patient-centered approach to healthcare has been found to increase efficiency of care, improve patient outcomes, increase HRQOL, reduce patient discomfort, and more adequately address patient concerns regarding healthcare. ${ }^{8,10}$

\section{Healthcare Disparities of LGBTQ+ Individuals}

Specific to the LGBTQ+ community, patientcentered care can mean the difference between an individual feeling comfortable enough to seek care and avoiding healthcare all together. A recent study published in the, Journal of Homosexuality, found that $37 \%$ of LGBTQ+ young adults do not disclose their identity to healthcare providers due to concerns over potential discrimination, internalized stigmas, fear of rejection, or a belief that health and LGBTQ+ identity are not related. 6 Apprehension towards seeking healthcare either in part by not feeling comfortable disclosing personal information, or in full by complete avoidance of seeking healthcare can be detrimental to the HRQOL of LGBTQ+ individuals.

Apprehension of LGBTQ+ individuals in seeking healthcare can be especially problematic for athletic trainers (ATs) particularly those practicing in traditional sports-focused settings at the professional, collegiate, or secondary school levels. While organizations such as the National Collegiate Athletic Association (NCAA) have made a concerted effort to create a more inclusive environment in sports, ideologies supporting homonegativity are still prevalent in all competitive levels of sport subculture in western society. ${ }^{11}$ Athletic trainers, especially in the aforementioned settings, are often the point of care for a disproportionately large number of patients. Given the close proximity with athletics, an ideology of homonegativity can either intentionally or unintentionally permeate into the clinic climate.

Consciously or unconsciously supporting and reinforcing a homonegative ideology in an

Copyright $\left({ }^{\circ}\right.$ by Indiana State University All rights reserved. ISSN Online 2577-8188 athletic training facility can substantiate lingering societal stigmas of LGBTQ+ individuals. ${ }^{9}$ Such an environment can prevent LGBTQ+ patients from seeking care from an $A T$, or disclosing pertinent information to the AT. Through being an active champion and advocate in identifying and addressing blatant bias and discrimination in athletic training facilities, ATs can focus on more patient-centered and equitable practice.

\section{Creating an Inclusive Facility Environment}

In order for a shift towards more equitable patient care to occur ATs must first take inventory of their own clinical practice, implicit biases, and communication styles in addition to the clinical setting in which they practice. One of the most difficult and perhaps tedious aspects of affecting change is awareness of not only systemic faults, but also personal weaknesses and areas of improvement. On an individual level, ATs must recognize their own verbal and non-verbal reactions when interacting with patients of diverse backgrounds. Inappropriate or incorrect language, body positioning, facial expression, or mannerisms, whether intentional or unintentional can cause the practitioner to appear as closed off or unwelcoming. ${ }^{12,13}$ These verbal and non-verbal cues, when intentionally exhibited towards underserved populations such as the LGBTQ+ community, are an example of demeaning micro aggressions. ${ }^{12,13}$ Adjustments to personal practice should be reinforced through the creation of facility or institution-wide policies that ensure discriminatory language, micro aggressions, and inappropriate behavior are addressed. ${ }^{12}$

In addition to an analysis of personal practice and athletic training facility policies, specific attention should be given to maintaining an inclusive clinic environment. In order to create a welcoming inclusive environment for LGBTQ+ individuals an athletic training facility can present visible representation supporting equality such as safe space ally stickers and posts of non-discrimination policies, and provide access to gender neutral restrooms as well as brochures or information addressing health concerns specific to the LGBTQ+ population. ${ }^{12,13}$ This development of an inclusive facility culture is especially pertinent for ATs, given the close relationship and proximity between practitioner and patient in the multiple 
different settings ATs practice. As such, it is important to create an inclusive facility culture before the first LGBTQ+ patient seeks care.

Creating and maintaining an equitable and culturally competent practice in athletic training is a difficult endeavor for any practitioner. In order to facilitate this development of a model practice, there are many training programs such as Safe Space and Ally training that provide sensitivity training to become cognizant of LGBTQ+ concerns, while simultaneously learning how to identify and address internal bias. ${ }^{5,12,13}$ These types of instructional trainings are an excellent way to bridge individual practitioner development, while at the same time addressing clinic or institutional areas of cultural weakness. $^{12,13}$

In addition to the aforementioned changes, another impactful adjustment that can be made to improve a facility's culture is the creation and utilization of inclusive documentation forms. While documentation forms, such as a pre-participation exam, are important for an athletic training facility to gather legal names and biological sex, it is equally important that the patient have the opportunity to self-report demographic information such as preferred name, gender, and gender identity. The most effective way to gather this information from the patient without being insensitive is by simply asking open-ended questions. ${ }^{13}$ This technique allows the patient to respond in whatever manner is appropriate, while also conveying that the practitioner respects the identity of the patient. Furthermore, utilizing an open-ended questioning technique allows the practitioner to collect pertinent demographic information, such as relationship status and appropriate pronouns, which are important when considering the HRQOL of LGBTQ+ patients and can be utilized in future patient interactions. ${ }^{7,12,13}$

An inclusive approach to patient interaction and documentation sets an important precedence that any patient, regardless of his-her-zir identity, is welcomed and will be treated with the upmost respect and fairness. Special attention, however, must be placed on avoiding ambivalent or neutral attitudes towards practicing in an equitable manner in order to maintain an inclusive athletic training facility environment. While not being an

Copyright $($ ) by Indiana State University All rights reserved. ISSN Online 2577-8188 active participant in creating an equitable practice might seem innocuous, inaction in addressing areas of weakness related to equity can be just as harmful to LGBTQ+ individuals as outright discrimination.

\section{Conclusion}

Major societal advancements in recent years have provided recognition and legal protections for LGBTQ+ individuals. These advancements, coupled with more exposure and access to competitive sports, have created a prominent need for ATs in all settings to ensure their practice is welcoming, inclusive, and culturally competent. Through the process of intentional reflection and analysis of athletic training facility culture, coupled with tangible changes, ATs can create an environment in which all patients, regardless of their identity, can feel comfortable seeking care.

\section{REFERENCES}

1. Lev Al, Sennott SL. Clinical work with LGBTQ parents and prospective parents. In: AE Goldber, KR Allen, eds. LGBT-Parent Families: Innovations in Research and Implications for Practice. New York, NY: Springer; 2013:241-60.

2. Masci D. Supreme Court's DOMA decision driving same-sex marriage efforts in states. Fact Tank: New in the Numbers: Pew Research Center, 2013.

http://www.pewresearch.org/facttank/2013/10/21/supreme-courts-domadecision-driving-same-sex-marriage-effortsin-states/. Accessed: May 1, 2018.

3. Frank N. The president's pleasant surprise: How LGBT advocates ended Don't Ask, Don't Tell. J Homosex. 2013;60(2-3):159- 213. https://doi.org/10.1080/00918369.2013 .744666

4. Institute of Medicine. The Health of Lesbian, Gay, Bisexual, and Transgender People: Building a Foundation for Better Understanding. Washington, DC: National Academies Press, 2011.

5. McKay B. Lesbian, gay, bisexual, and transgender health issues, disparities, and information resources. Med Ref Serv Q. 
$2011 ; 30(4): 393-401$.

https://doi.org/10.1080/02763869.2011 .608971

6. Rossman K, Salamanca P, Macapagal K. A qualitative study examining young adults' experiences of disclosure and nondisclosure of LGBTQ identity to health care providers. J Homosex. 2017;64(10):1390-410. https://doi.org/10.1080/00918369.2017 .1321379

7. Weiner SJ, Schwartz A, Sharma G, et al. Patient-centered decision making and health care outcomes: an observational study. Ann Intern Med. 2013;158(8):573- 79. https://doi.org/10.7326/0003-4819158-8-201304160-00001

8. Epstein RM, Street RL. The values and value of patient-centered care: Ann Fam Med. 2011 ;9(2)100-103. https://doi.org/10.1370/afm.1239

9. Snyder AR, Parsons JT, Valovich McLeod TC, Curtis Bay R, Michener LA, Sauers EL. Using disablement models and clinical outcomes assessment to enable evidence based athletic training practice, Part I: Disablement models. J Athl Train. 2008;43(4):428-36. https://doi.org/10.4085/1062-605043.4.428

10. Oates J, Weston WW, Jordan J. The impact of patient-centered care on outcomes. Fam Pract. 2000;49(9):796- 804.

11. Anderson E, Magrath R, Bullingham R. Out in Sport: The Experiences of Openly Gay and Lesbian Athletes in Competitive Sport: New York, NY: Routledge; 2016.

12. McClain Z, Hawkins LA, Yehia BR. Creating welcoming spaces for lesbian, gay, bisexual, and transgender (LGBT) patients: An evaluation of the health care environment. J Homosex. 2016;63(3):38793. https://doi.org/10.1080/00918369.201 6.1124694

13. McNair RP, Hegarty K. Guidelines for the primary care of lesbian, gay, and bisexual people: a systematic review. Ann Fam Med. 2010;8(6):533-41. https://doi.org/10.1370/afm.1173 